\title{
LIST OF PAPERS READ, BUT NOT PUBLISHED
}

The amplitude interferometry program
D. Currie

CCD astrometry at KPNO and USNO-Flagstaff

C. C. Dahn and D. Monet

Reduction of VLBI observations

$$
\text { T. A. Herring }
$$

Radio interferometric astrometry: techniques and results

K. Johnston

The astrometric applications of interferometry in the visual and infrared regions of the spectrum

C. H. Townes 